\title{
FENÓLICOS E CAROTENÓIDES TOTAIS EM PITANGA
}

\author{
Vera Lúcia Arroxelas Galvão de Lima ${ }^{1 *}$; Enayde de Almeida Mélo'; Daisyvângela E. da Silva \\ Lima $^{2}$ \\ ${ }^{1}$ Depto. de Ciências Domésticas - UFRPE - R. Dom Manoel de Medeiros s/n, Dois Irmãos - CEP: 52171-900 - \\ Recife, $P E$. \\ 'Graduanda em Economia Doméstica - UFRPE. \\ *Autor correspondente <veraarroxelas@hotmail.com>
}

RESUMO: Em decorrência de uma ampla diversidade genética, a pitanga (Eugenia uniflora L.) apresenta cor que varia do alaranjado até o vermelho escuro quase negro. Em Pernambuco, no entanto, é comum encontrála com coloração laranja-avermelhada. Como muitos fitoquímicos presentes em frutos exibem propriedades antioxidantes, com destaque para carotenóides e compostos fenólicos, e frente a escassez de informações sobre seus teores em pitanga, objetivou-se quantificá-los nas seleções cujos frutos apresentam coloração vermelha e roxa. Assim, foram determinados espectrofotometricamente os teores de carotenóides e fenólicos totais nos frutos destas seleções nos estádios maduro e semi-maduro. Na pitanga roxa madura e em sua película também foram determinados os teores de antocianinas, flavonóis e carotenóides totais. Os compostos fenólicos e os carotenóides totais na pitanga roxa madura encontram-se em maiores teores do que na pitanga vermelha no mesmo estádio de maturação. A seleção roxa exibiu quantidade significante de antocianinas, cujo teor foi mais elevado no fruto maduro do que no semi-maduro. Os carotenóides, antocianinas e flavonóis encontram-se mais concentrados na película do que na polpa deste fruto maduro. A presença de antocianinas, flavonóis e carotenóides totais na pitanga roxa fazem deste fruto uma fonte promissora de compostos antioxidantes cujo cultivo deveria ser estimulado.

Palavras-chave: Eugenia uniflora, antocianinas, fenólicos totais, carotenóides totais, estádios de maturação

\section{TOTAL PHENOLICS AND CAROTENOIDS IN SURINAM CHERRY}

\begin{abstract}
Because of the genetic diversity, fruits of Surinam cherry (Eugenia uniflora L.) show colours that range from orange to deep, almost dark red. However, in Pernambuco, Brazil, it is common to find this fruit with an orange-red colour. Many phytochemicals in fruits have antioxidant properties with emphasis on phenolic compounds and carotenoids. Since the information on these phytochemicals in surinam cherry is scarce, the aim of this research was to analyse them in fruits which show red and purple colours. Half-mature and mature fruits were used to evaluate these phytochemicals. In purple surinam cherries and their peel, total anthocyanins, flavonols and carotenoids were determined. Phenolic compounds and carotenoids had higher concentration in purple mature cherries than in red ones, at the same stage of maturation. Purple cherries showed significant quantity of anthocyanin with values higher in mature fruits than in the half-mature. Total carotenoids, anthocyanins and flavonols were higher in the peel than in the pulp of mature fruits. The anthocyanin, flavonol and carotenoid contents in purple surinam cherry make this fruit a promising source of antioxidant compounds. For this reason the cultivation of this crop should be encouraged.
\end{abstract}

Key words: Eugenia uniflora, anthocyanins, total phenolics, total carotenoids, maturity stages

\section{INTRODUÇÃO}

Dentre as mais conhecidas espécies de Eugenia, a pitanga também conhecida por "Brazilian cherry" é encontrada em vários países uma vez que se adapta favoravelmente às diferentes condições climáticas e edáficas. Na Espanha é chamada de "cereza de cayena", na Venezuela de "pedanga", em El Salvador de "guinda", na Argentina de "ñanga-piré", na Colômbia de "cereza quadradra" (Morton, 1987). O fruto é uma baga globosa, com sete a dez sulcos longitudinais de 1,5 a $5,0 \mathrm{~cm}$ de diâmetro, coroado com sépalas persistentes que possui aroma característico intenso e sabor doce e ácido. No processo de maturação, o epicarpo passa de verde para amarelo, alaranjado, vermelho, vermelhoescuro, podendo chegar até quase negro (Bezerra et al., 2000). No Brasil não se conhecem variedades perfeitamente definidas de pitangueiras e em Pernambuco, é comum encontrar pitanga de coloração variando de alaranjada a avermelhada (Lederman et al., 1992).

O consumo de frutas e hortaliças tem aumentado principalmente em decorrência do seu valor nutritivo e efeitos terapêuticos. Estes alimentos contêm diferentes fitoquímicos, muitos dos quais possuem propriedade antioxidante que pode estar relacionada com o retardo do envelhecimento e a prevenção de certas doenças. Estudos têm demonstrado que outros compostos antioxidantes, além dos bem conhecidos $\beta$-caroteno, vitamina $C$ e vitamina $E$, têm contribuído para a total capacidade antioxidante desses vegetais (Wang et al., 1996; Rice-Evans et al., 1996). Em recentes pesquisas foi evidenciado que compostos fenólicos exibem ação antioxidante. Pearson, et al. (1999) demonstraram que 
os fenólicos presentes em suco comercial e extrato fresco de maçãs (casca, polpa e fruta inteira) inibiram, in vitro, a oxidação de LDL humana. A atividade antioxidante apresentada por vários vegetais, incluindo frutos, folhas, sementes e plantas medicinas, está correlacionada ao seu teor de compostos fenólicos totais (Velioglu et al., 1998). Os compostos fenólicos são responsáveis pela atividade antioxidante de diversos vegetais (Kähkönen et al., 1999).

Dentre os compostos fenólicos com propriedade antioxidante, destacam-se os flavonóides que quimicamente, englobam as antocianinas e os flavonóis. As antocianinas são pigmentos solúveis em água, amplamente difundidas no reino vegetal e conferem as várias nuanças de cores entre laranja, vermelha e azul encontradas em frutas, vegetais, flores, folhas e raízes (Francis, 1989). Os flavonóis são pigmentos de cores branca ou amarela clara encontrados nesses alimentos. Os últimos pigmentos citados são importantes por atuarem na co-pigmentação das antocianinas (Bobbio \& Bobbio,1995). Atualmente, existe uma tendência mundial em usar pigmentos naturais como corantes para alimentos e entre eles destacam-se as antocianinas. Esse interesse é também influenciado pelas observações promissoras de seu potencial benéfico à saúde decorrente de sua ação antioxidante (Espín et al., 2000, Wang et al., 1997).

Considerando a escassez de informações sobre fitoquímicos na pitanga este trabalho teve como objetivo quantificar os teores de compostos fenólicos e de carotenóides totais presentes nesta fruta.

\section{MATERIAL E MÉTODOS}

Três lotes distintos com $200 \mathrm{~g}$ de duas seleções de pitanga (roxa e vermelha) (Figura 1), procedentes de um plantio, localizado em Paratibe- PE, colhidas em dois estádios de maturação, foram utilizadas para determinar os teores de fenólicos e carotenóides totais. O grau de cor da película foi utilizado para classificação dos frutos quanto ao estádio de maturação. Para a seleção roxa, os frutos considerados semi-maduros apresentavam coloração vermelha-arroxeada e os maduros coloração roxa intensa. Os frutos semimaduros e maduros da seleção vermelha possuíam coloração vermelha-alaranjada e vermelha intensa, respectivamente.

No Laboratório de Análises Físico-químicas e Sensorial de Alimentos - DCD/UFRPE, os frutos foram processados para obtenção da polpa utilizando uma centrífuga doméstica. A extração dos carotenóides totais da polpa obtida foi efetuada pelo método descrito por Rodriguez-Amaya (1999) e a para sua quantificação foi utilizado o espectro de absorção registrado no comprimento de onda de $450 \mathrm{~nm}$ e a expressão matemática descrita por Gross (1987), considerando o coeficiente de absorção de 2500 . Os fenólicos totais

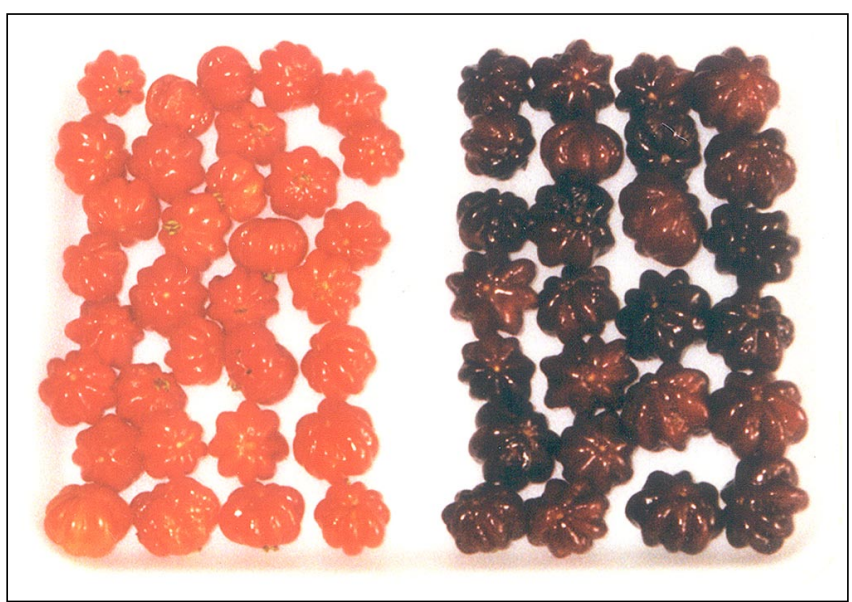

Figura 1 - Pitangas maduras das seleções vermelha e roxa.

foram determinados de acordo com a metodologia descrita por Tsimidou et al. (1992) usando o reagente de Folin-Ciocalteu tendo a catequina como padrão. Todas as determinações foram efetuadas em triplicata e os resultados submetidos à análise estatística, teste $\mathrm{t}$, a $5 \%$, utilizando o programa estatístico "Minitab -10 for Windows".

Na polpa da pitanga roxa, dos dois estádios de maturação, foram determinados os teores de antocianinas e flavonóis totais através do método espectrofotométrico de Lees \& Francis (1972), com as modificações descritas por Lima et al. (2000). A película da pitanga roxa madura foi retirada com o auxílio de uma faca afiada de aço inox e também submetida às determinações de carotenóides, antocianinas e flavonóis totais utilizando os métodos citados acima.

\section{RESULTADOS E DISCUSSÃO}

Ao comparar os teores de fenólicos totais dos frutos das duas seleções no mesmo estádio de maturação, observa-se que estes fitoquímicos nas pitangas semi-maduras não apresentaram diferença (Tabela 1). O teor de compostos fenólicos na pitanga roxa madura foi maior do que na vermelha do mesmo estádio de maturação. A cor característica da seleção roxa decorre da presença de antocianinas, o que justifica o elevado teor de fenólicos nesta seleção, tendo em vista que pigmentos antociânicos são considerado compostos fenólicos (Lima et al., 2000). Durante o amadurecimento de ameixas de quatro cultivares, houve um aumento no teor de fenólicos totais (Nascimento et al., 1990). Este resultado corrobora os encontrados neste estudo uma vez que também as antocianinas são os pigmentos responsáveis pela cor vermelha das ameixas (Draetta et al., 1985).

Os teores de antocianinas e flavonóis totais da

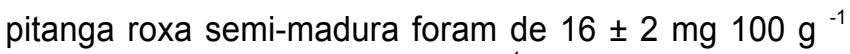
e $14 \pm 2 \mathrm{mg}$ de quercetina $100 \mathrm{~g}^{-1}$, respectivamente. Ao comparar estes valores com os apresentados na Tabela 2 observa-se que o fruto maduro apresentou maiores 
teores destes fitoquímicos. Lima et al. (2000) encontraram para este fruto maduro, valores de antocianinas e flavonóis totais $\left(22,50 \mathrm{mg} 100 \mathrm{~g} \mathrm{~g}^{-1} \mathrm{e} \mathrm{13,93}\right.$ mg de quercetina $100 \mathrm{~g}^{-1}$, respectivamente) semelhantes aos determinados neste estudo. Em "cranberries" estes dois pigmentos, também, encontravam-se em maior concentração no fruto maduro do que no semi-maduro (Lees \& Francis, 1971). O maior teor de antocianinas no estádio maduro é justificado pela síntese destes pigmentos que ocorre durante o seu amadurecimento, atingindo o valor máximo no fruto completamente maduro (Gross, 1987). Em cereja ácida também foi evidenciado um aumento gradativo no teor de antocianinas totais durante o amadurecimento do fruto. No estádio muito imaturo esta cereja apresentou teor de $2,0 \mathrm{mg} 100 \mathrm{~g}^{-1}$ enquanto que no estádio maduro atingiu o valor de 43,6 mg $100 \mathrm{~g}^{-1}$ (Dekazos, 1970).

Considerando o peso fresco do fruto, o teor de antocianinas e flavonóis totais da pitanga roxa madura foi de $0,03 \%$ e $0,02 \%$, respectivamente, enquanto que na película do fruto foi de $0,42 \%$ para as antocianinas totais e de $0,12 \%$ para os flavonóis totais. Assim, estes dois fitoquímicos presentes neste fruto maduro estão mais concentrados na película do que em sua polpa. Dekazos (1970) quantificando o teor de antocianinas totais em cereja ácida madura (fruto e película), evidenciou que este pigmento também encontrava-se em maior proporção na película $(0,4 \%)$ do que no fruto (aproximadamente $0,05 \%$ ). $\mathrm{Em}$ alguns frutos as antocianinas estão localizadas

Tabela 1 - Teores de compostos fenólicos e carotenóides totais em duas seleções de pitanga.

\begin{tabular}{lcccc}
\hline & \multicolumn{2}{c}{ Pitanga roxa } & \multicolumn{2}{c}{ Pitanga vermelha } \\
\cline { 2 - 5 } Determinação & Madura & $\begin{array}{c}\text { Semi } \\
\text { madura }\end{array}$ & Madura & $\begin{array}{c}\text { Semi } \\
\text { madura }\end{array}$ \\
\hline $\begin{array}{l}\text { Fenólicos totais } \\
\left(\mathrm{mg} 100 \mathrm{~g}^{-1}\right)^{*}\end{array}$ & $325 \pm 24$ & $257 \pm 12^{* *}$ & $257 \pm 3$ & $252 \pm 4^{* *}$ \\
$\begin{array}{l}\text { Carotenó ides } \\
\text { totais }\left(\mu \mathrm{g} \mathrm{g}^{-1}\right)^{* * *}\end{array}$ & $111 \pm 2$ & $98 \pm 1$ & $104 \pm 0$ & $79 \pm 1$ \\
\hline
\end{tabular}

${ }^{*} \mathrm{mg}$ em equivalente de catequina $100 \mathrm{~g}^{-1}$

** os valores não apresentam diferença significativa - Teste $t$ Student

${ }^{* * *} \mu \mathrm{g}$ em equivalente de $\beta$-caroteno $\mathrm{g}^{-1}$

Tabela 2 - Teores de antocianinas, flavonóis e carotenóides totais da pitanga roxa madura.

\begin{tabular}{lcc}
\hline Determinação & Película & Polpa \\
\hline $\begin{array}{l}\text { Antocianinas totais } \\
\left(\mathrm{mg} 100 \mathrm{~g}^{-1}\right)\end{array}$ & $420 \pm 0$ & $26 \pm 0$ \\
$\begin{array}{l}\text { Flavonó is totais } \\
\left(\mathrm{mg} 100 \mathrm{~g}^{-1}\right)^{*}\end{array}$ & $120 \pm 16$ & $18 \pm 0$ \\
$\begin{array}{l}\text { Carotenóides totais } \\
\left(\mu \mathrm{g} \mathrm{g}^{-1}\right)^{* *}\end{array}$ & $750 \pm 9$ & $111 \pm 2$ \\
\hline
\end{tabular}

${ }^{*} \mathrm{mg}$ de quercetina $100 \mathrm{~g}^{-1}$

${ }^{* *} \mu \mathrm{g}$ em equivalente de $\beta$-caroteno $\mathrm{g}^{-1}$ principalmente na película, com é o caso de ameixa e uva, e em outros este pigmento encontra-se distribuído em todo o fruto, a exemplo do morango, "currant" e "raspberry" (Gross, 1987).

A pitanga roxa por apresentar uma quantidade significante de antocianinas exibe uma cor diferenciada, atrativa e pouco comum. Lima et al.(2000) ao realizarem avaliação sensorial do suco de pitanga roxa e vermelha, através do Teste de Preferência - Comparação Pareada, evidenciaram diferença na preferência dos degustadores pelo suco de pitanga roxa. A cor peculiar dessa pitanga certamente influenciou na avaliação dos provadores. A presença de antocianinas além de contribuir para a preferência desse fruto, possui potente propriedade antioxidante como foi evidenciada por Espín et al. (2000), Kalt et al. (2000), Satué-Gracia et al. (1997) e Wang et al. (1997).

Nas duas seleções, o teor de carotenóides totais do fruto maduro foi maior do que no semi-maduro, entretanto a quantidade deste fitoquímico foi significativamente mais elevada na pitanga roxa do que na vermelha (Tabela 1). O valor médio referente ao conteúdo total de carotenóides em pitanga vermelha determinado por Cavalcante (1991) foi de $225,9 \mathrm{mg} \mathrm{g}^{-1}$ o qual foi superior ao encontrado neste estudo. Para determinar este valor o autor acima citado considerou o somatório dos teores dos pigmentos fracionados que foram quantificados utilizando $\lambda_{\text {max }}$ e o coeficiente de absortividade específico para cada fração. Neste estudo, como não foi efetuado o fracionamento dos carotenóides, a sua quantificação foi determinada considerando $\circ \lambda_{\text {máx }}$ e o coeficiente de absortividade recomendado para carotenóides não fracionados, o que possivelmente contribuiu para a quantificação de um menor teor. Além disso, a composição química de frutos pode ser afetada por diversos fatores, a exemplo do grau de maturação, condições climáticas, edáficas e cultivares analisados, entre outros (Harris, 1977). Assim, a influência destes fatores poderia também explicar a discrepância evidenciada entre os valores determinados nestes dois estudos.

O teor de carotenóides totais aumenta durante o amadurecimento, momento em que a carotenogênese é intensificada, a exemplo da manga (John et al., 1970), laranja (Rotstein et al., 1972), mamão (Wilberg \& Rodriguez-Amaya, 1995) e carambola (Gross et al., 1983). Em outros frutos, nos quais a cor do estádio maduro é devido à presença de antocianinas, a exemplo de morango (Woodward, 1972) e "red currant" (Gross 1982/83), o teor de carotenóides diminui com o amadurecimento. Na pitanga roxa foi observado um comportamento diferente: o fruto maduro apresentou teores de antocianinas e carotenóides totais maiores do que no semi-maduro. Assim, apesar da cor característica deste fruto ser devida aos pigmentos antociânicos, o teor de carotenóides totais não diminuiu com o seu amadurecimento. 
A distribuição dos carotenóides em frutos é extremamente complexa e sujeita à consideráveis variações (Gross, 1987). Alguns pesquisadores encontraram que os carotenóides são geralmente mais concentrados na película do que na polpa de alguns frutos. Em abacates, por exemplo, o teor de carotenóides totais na polpa variou de $10-14 \mu \mathrm{g} \mathrm{g}^{-1}$ de peso fresco enquanto na película o teor foi de $40 \mu \mathrm{g} \mathrm{g}^{-1}$ de peso fresco (Gross et al., 1973). Na pitanga roxa madura estes compostos encontram-se, também, mais concentrados na película do que na polpa (Tabela 2).

\section{CONCLUSÕES}

As seleções de pitanga estudadas apresentam consideráveis teores de fenólicos e carotenóides totais. A seleção roxa no estádio maduro destaca-se por apresentar maiores teores destes fitoquímicos. A presença de antocianinas aliada aos teores de flavonóis e carotenóides totais fazem deste fruto uma fonte promissora de compostos antioxidantes cujo cultivo deveria ser estimulado.

\section{AGRADECIMENTOS}

À UFRPE e ao PIBIC-CNPq/UFRPE pelo auxílio financeiro concedido na realização desta pesquisa.

\section{REFERÊNCIAS BIBLIOGRÁFICAS}

BEZERRA, J.E.F.; SILVA JR., J.F. da; LEDERMAN, I.E. Pitanga (Eugenia uniflora L.) Jaboticabal: Funep, 2000. 30p. (Série Frutas Nativas, 1)

BOBBIO, P.A.; BOBBIO, F.O. Introdução à química de alimentos. 2.ed. São Paulo: Varela, 1995. 223p.

CAVALCANTE, M.L. Composição de carotenóides e valor de vitamina A na pitanga (Eugenia uniflora) e acerola (Malpighia glabra.). Rio de Janeiro, 1991. 73p. Dissertação (Mestrado) - Instituto de Nutrição, Universidade Federal do Rio de Janeiro.

DRAETTA, I.S.; IADEROZA, M.; BALDINI, V.L.S.; FRANCIS, F.J. Antocianinas de ameixa (Prunus salicina L.) Ciência e Tecnologia de Alimentos, v.5, p.31-38, 1985.

DEKAZOS, E.D. Quantitative determination of anthocyanin pigments during the maturation and ripening of red tart cherries. Journal of Food Science, v.35, p.242-244, 1970

ESPÍN, J.C.; SOLER-RIVAS, C.; WICHERS, H.J.; GARCÍA-VIGUERA, C. Anthocyanin-based natural colorants: a new source of antiradical activity for foodstuff. Journal of Agricultural and Food Chemistry, v.48, p.15881592, 2000.

FRANCIS, F.J. Food colorants: anthocyanins. Critical Reviews in Food Science and Nutrition, v.28, p.273-314, 1989.

GROSS, J.; GABAI, M.; LIFSHITZ, A.; SKLARZ, B. Carotenoids in pulp, peel and leaves of Persea americana. Phytochemistry, v.12, p.2259-2263, 1973.

GROSS, J. Pigments in fruits. London: Academic Press, 1987. 303p.

GROSS, J. Chlorophyll and carotenoid pigments in Ribes fruits. Scientia Horticulturae, v.18, p.131-136, 1982/83.
GROSS, J.; IKAN, R.; ECKHARDT, G. Carotenoids of the fruit of Averrhoa carambola. Phytochemistry, v.22, p.1479-1481,1983.

HARRIS, R.S. Effects of agricultural practices on foods of plant origin. In: HARRIS, R.S.; KARMAS, E. (Ed.) Nutritional evaluation of food processing. Connecticut: Avi Publishing, 1977. cap.6, p.33-57.

JOHN, J.; SUBBARAYAN, C.; CAMA, H.R. Carotenoids in 3 stages of ripening of mango. Journal of Food Science, v.35, p.262-265, 1970.

KÄHKÖNEN, M.P.; HOPIA, A.I.; VUORELA, H.J.; RAUHA, J.-P.; PIHLAJA, K.; KUJALA, T.S.; HEINONEN, M. Antioxidant activity of plant extracts containing phenolic compounds. Journal of Agricultural and Food Chemistry, v.47, p.3954-3962, 1999.

KALT, W.; McDONALD, J.E.; DONNER, H. Anthocyanins, phenolics and antioxidant capacity of processed lowbush blueberry products. Journal of Food Science, v.65, p.390-393, 2000.

LEDERMAN, I.E.; BEZERRA, J.E.F.; CALADO, G. A pitangueira em Pernambuco. Recife: Secretaria de Agricultura, Empresa Pernambucana de Pesquisa Agropecuária- IPA. 1992. 20p. (Documentos, 19)

LEES, D.H.; FRANCIS, F.J. Quantitative methods for anthocyanis. 6. Flovonols and anthocyanins in cranberries. Journal of Food Science, v.36, p.10561060, 1971.

LEES, D.H.; FRANCIS, F.J. Standardization of pigment analyses in cranberries. HortScience, v.7, p.83-84, 1972.

LIMA, V.L.A.G de; MÉLO, E. de A.; LIMA, L. dos S.; NASCIMENTO, P.P. do. Caracterização físico-química e sensorial de pitanga roxa. Revista Brasileira de Fruticultura, v.22, p.382-385, 2000.

MORTON, J.F. Fruits of warm climates. Miami: AgScience, 1987. 559p.

NASCIMENTO, L.M. do; CHITARRA, A.B.; CHITARRA, M.I.F. Fisiologia pós colheita de frutos de quatro cultivares de ameixeira (Prunus sp.) armazenados em diferentes condições. Coletânea do Instituto de Tecnologia de Alimentos, v.20, p.155-171, 1990.

PEARSON, D.A.; TAN, C.H.; GERMAN, J.B.; DAVIS, P.A.; GERSHWIN, M.E. Apple juice inhibits human low density lipoprotein oxidation. Life Sciences, v.64, p.1913-1920, 1999.

RICE-EVANS, C.A.; MILLER, N.J.; PAGANGA, G. Structure-antioxidant activity relationships of flavonoids and phenolic acids. Free Radical Biology \& Medicine, v.20, p.933-956, 1996.

RODRIGUEZ-AMAYA, D.B. A guide to carotenoid analysis in foods. Washington: ILSI Press, 1999. 64p.

ROTSTEIN, A.; GROSS, J.; LIFSHITZ, A. Changes in the pulp carotenoid pigments of the ripening Shamouti orange. Lebensmittel - Wissenschaft und Technologie, v.5, p.140-143, 1972.

SATUÉ-GRACIA, M.T.; HEINONEN, M.; FRANKEL, E.N. Anthocyanins as antioxidants on human low-density lipoprotein and lecithin-liposome systems. Journal of Agricultural and Food Chemistry, v.45, p.33623367, 1997.

TSIMIDOU, M.; PAPADOPOULOS, G.; BOSKOU, D. Phenolic compounds and stability of virgin olive oil - Part I. Food Chemistry, v.45, p.141-144, 1992.

VELIOGLU, Y.S.; MAZZA, G.; GAO, L.; OOMAH, B.D. Antioxidant activity and total phenolics in selected fruits, vegetables, and grain products. Journal of Agricultural and Food Chemistry, v.46, p.4113-4117, 1998.

WANG, H.; CAO, G.; PRIOR, R.L. Total antioxidant capacity of fruits. Journal of Agricultural and Food Chemistry, v.44, p.701-705, 1996.

WANG, H.; CAO, G.; PRIOR, R. L. Oxigen radical absorbing capacity of anthocyanins. Journal of Agricultural and Food Chemistry, v.45, p.304309, 1997.

WILBERG, V.C. ; RODRIGUEZ-AMAYA, D.B. HPLC quantitation of major carotenoids of fresh and processed guava, mango and papaya. Lebensmittel - Wissenschaft und Technologie, v.28, p.474-480, 1995. WOODWARD, J.R. Physical and chemical changes in developing strawberry fruits. Journal of the Science of Food and Agriculture, v.23, p.465473, 1972

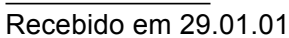

